\title{
Optimized Schwarz Waveform Relaxation Methods: A Large Scale Numerical Study
}

\author{
Martin J. Gander ${ }^{1}$, Loïc Gouarin ${ }^{2}$, and Laurence Halpern ${ }^{2}$ \\ 1 Section de mathématiques, Université de Genève, 2-4 rue du Lièvre, CP 64, \\ CH-1211 Genève 4, Switzerland. Martin.Gander@unige.ch \\ 2 Laboratoire Analyse, Géométrie et Applications Université Paris XIII, 99 \\ Avenue J.-B. Clément, 93430 Villetaneuse, France. \\ \{gouarin|halpern\}@math.univ-paris13.fr
}

\section{Introduction}

Schwarz waveform relaxation methods are naturally parallel methods to solve evolution problems. They are based on a decomposition of the physical domain into overlapping subdomains, and a decomposition of the time domain into time windows. On each time window, one then solves the original time dependent problem, and a subdomain iteration like in the classical Schwarz method, but now in space-time, is used in order to obtain a converged solution on the present time window. Only after convergence on the time window is the next time window treated by the algorithm. This type of algorithm was first proposed in [2], and analyzed in [8] and independently in [10]. Optimized Schwarz waveform relaxation methods were introduced in [7] to obtain more effective space-time iterative methods, compared to the classical variants, and the associated optimization problem was studied in [6] for the case of Robin conditions, and in [1] for higher order transmission conditions, see also [12]. Our extensive numerical experiments (a summary is given in Table 1), reveal that, while the theoretical parameters are asymptotically optimal, the performance can be substantially improved using a more accurate estimate for the constant. We show in this paper that this difference can be put on a theoretical foundation by taking into account geometric parameters from the decomposition. We illustrate the improved performance with the new parameters by numerical experiments, and also study numerically the dependence of the parameter on the number of subdomains.

\section{Optimized Schwarz Waveform Relaxation}

We study the optimized Schwarz waveform relaxation algorithm for the advection reaction diffusion equation in $\Omega \subset \mathbb{R}^{2}$, 


$$
\mathcal{L} u:=u_{t}+\boldsymbol{a} \cdot \nabla u-\nu \Delta u+b u=f, \quad \text { in } \Omega \times(0, T),
$$

where $\nu>0, b \geq 0$ and $\boldsymbol{a}=(a, c)^{T}$. In order to describe the Schwarz waveform relaxation algorithm, we decompose the domain into two, possibly overlapping subdomains $\Omega_{1}$ and $\Omega_{2}$, with interfaces $\Gamma_{1}=\partial \Omega_{1} \cap \Omega_{2}$ and $\Gamma_{2}=\partial \Omega_{1} \cap \Omega_{2}$. The algorithm for this two subdomain decomposition calculates then for $n=$ $1,2, \ldots$ the iterates $\left(u_{1}^{n}, u_{2}^{n}\right)$ defined by

$$
\begin{aligned}
& \mathcal{L} u_{1}^{n}=f \quad \text { in } \Omega_{1} \times(0, T), \quad \mathcal{L} u_{2}^{n}=f \quad \text { in } \Omega_{2} \times(0, T), \\
& u_{1}^{n}(\cdot, \cdot, 0)=u_{0} \quad \text { in } \Omega_{1}, \quad u_{2}^{n}(\cdot, \cdot, 0)=u_{0} \quad \text { in } \Omega_{2} \text {, } \\
& \mathcal{B}_{1} u_{1}^{n}=\mathcal{B}_{1} u_{2}^{n-1} \text { on } \Gamma_{1} \times(0, T), \quad \mathcal{B}_{2} u_{2}^{n}=\mathcal{B}_{2} u_{1}^{n-1} \text { on } \Gamma_{2} \times(0, T),
\end{aligned}
$$

where $\mathcal{B}_{1}$ and $\mathcal{B}_{2}$ are linear operators in space and time, possibly pseudodifferential, and an initial guess $\mathcal{B}_{2} u_{1}^{0}(0, \cdot, \cdot)$ and $\mathcal{B}_{1} u_{2}^{0}(L, \cdot, \cdot), t \in(0, T)$, needs to be provided.

\section{Theoretical Results}

There are many different choices for the operators $\mathcal{B}_{j}$. The identity leads to the classical Schwarz waveform relaxation method, and zeroth or higher order conditions lead to optimized variants, see for example [6] and [1]. We study here in detail the case where the transmission operators are

$$
\mathcal{B}_{1}:=\partial_{x}-\frac{a-p}{2 \nu} \quad \mathcal{B}_{2}:=\partial_{x}-\frac{a+p}{2 \nu} .
$$

Using Fourier analysis, and a decomposition of the domain $\Omega=\mathbb{R}^{2}$ into two half spaces $\Omega_{1}=(-\infty, L) \times \mathbb{R}$ and $\Omega_{2}=(0, \infty) \times \mathbb{R}$, see for example [6], one can obtain the convergence factor of algorithm (2),

$$
\rho(\omega, k, p)=\frac{z-p}{z+p} e^{-\frac{L z}{2 \nu}}
$$

where $z:=\sqrt{x_{0}^{2}+4 \nu^{2} k^{2}+4 i \nu(\omega+c k)}$ (standard branch of the square root with positive real part), $x_{0}^{2}:=a^{2}+4 \nu b$, and $k$ and $\omega$ are the Fourier variables in space and time. Computing on a grid, we assume that $k_{\max }=\frac{\pi}{h}$ where $h$ is the local mesh size in $x$ and $y$, and $\omega_{\max }=\frac{\pi}{\Delta t}$, and that we also have estimates for the lowest frequencies $k_{\min }$ and $\omega_{\min }$ from the geometry, see for example [4], or for a more precise analysis see [5].

Defining $D:=\left\{(\omega, k), \omega_{\min } \leq|\omega| \leq \omega_{\max }, k_{\min } \leq|k| \leq k_{\max }\right\}$, the parameter $p^{*}$ which gives the best convergence rate is solution of the best approximation problem

$$
\inf _{p \in \mathbb{C}} \sup _{(\omega, k) \in D}|\rho(\omega, k, p)|=\sup _{(\omega, k) \in D}\left|\rho\left(\omega, k, p^{*}\right)\right|=: \delta^{*} .
$$

In what follows, we will use 


$$
\bar{k}:=\frac{|c|\left(\sqrt{\left(|c|^{2}+x_{0}^{2}\right)^{2}+16 \nu^{2} \omega_{\min }^{2}}-|c|^{2}-x_{0}^{2}\right)}{8 \nu^{2} \omega_{\min }} .
$$

By a direct calculation, we see that $0 \leq \bar{k}|c| \leq \omega_{\text {min }}$, and we define the function

$$
\varphi(k, \xi):=2 \sqrt{2} \sqrt{\sqrt{\left(x_{0}^{2}+4 \nu^{2} k^{2}\right)^{2}+16 \nu^{2} \xi^{2}}+x_{0}^{2}+4 \nu^{2} k^{2}},
$$

and the constant

$$
A= \begin{cases}\varphi\left(\bar{k},-\omega_{\min }+|c| \bar{k}\right) & \text { if } k_{\min } \leq \bar{k} \\ \varphi\left(k_{\min },-\omega_{\min }+|c| k_{\min }\right) & \text { if } \bar{k} \leq k_{\min } \leq \frac{1}{|c|} \omega_{\min } \\ \varphi\left(k_{\min }, 0\right) & \text { if } k_{\min } \geq \frac{1}{|c|} \omega_{\min } .\end{cases}
$$

We assume that the mesh sizes in time and space are related either by $\Delta t=$ $C_{h} h$, or $\Delta t=C_{h} h^{2}$. The following theorem gives the asymptotic value of the best parameter $p^{*}$ in the case of no overlap, $L=0$, for the general case where the geometric parameters $k_{\min }$ and $\omega_{\min }$ are non zero. This is an important generalization of the result from [11], where $k_{\min }=\omega_{\min }=0$. The proof of this result is beyond the scope of this short paper, and will appear elsewhere.

Theorem 1. For $h$ small, the best approximation problem (5) has a unique solution $p^{*}$, which is given asymptotically by

$$
p^{*} \sim \sqrt{\frac{A}{B h}}, \quad \delta^{*}=1-\sqrt{A B h}+O(h),
$$

where $A$ is defined in (7), and

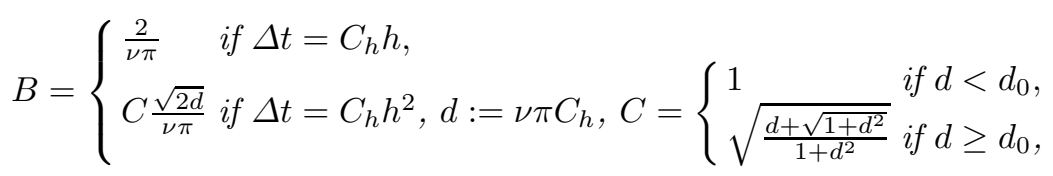

where $d_{0} \approx 1.543679$ is the unique real root of the polynomial $d^{3}-2 d^{2}+2 d-2$.

We take a closer look at two special cases:

1. If $k_{\min }=\omega_{\min }=0$, all three cases for $A$ in (7) coincide, since $\bar{k}=0$, and the constant $A$ simplifies to

$$
A=4 x_{0},
$$

and we find the special case analyzed in [11].

2. For the heat equation, $a=0, b=0, c=0, \nu=1$, and if $k_{\min }$ and $\omega_{\min }$ do not both vanish simultaneously, we also obtain $\bar{k}=0$, and

$$
A=4 \sqrt{2\left(\sqrt{k_{\min }^{4}+\omega_{\min }^{2}}+k_{\min }^{2}\right)}
$$

the special case analyzed in [14]. 


\section{Numerical Experiments}

We discretize (1) with $\boldsymbol{a}:=(1,1), \nu=0.1$ and $c=0$ on $\Omega=(0,1.2) \times(0,1.2)$ using P1 finite elements on a regular triangular mesh with $h=\frac{1}{100}$, and backward Euler with $\Delta t=\frac{1}{400}$. We simulate directly the homogeneous error equations, start the iteration with a random initial guess, and stop when the residual becomes smaller than $10^{-6}$. We do not use a coarse grid.

Table 1 shows iteration numbers for the new optimized parameter $p^{*}$ from Theorem 1, compared to the old one from [11] given in (8), and the parameters which work numerically best (found using a multi directional simplex method, see [13]). We provide iteration numbers both for the algorithm used as an iterative solver, and as a preconditioner for GMRES. The left column gives

\begin{tabular}{|c|ccc|ccc|}
\hline & \multicolumn{3}{|c|}{ Iterative } & \multicolumn{3}{c|}{ GMRES } \\
\hline decomposition & old & new & numerical & old & new & numerical \\
\hline \hline $2 \times 1$ & 271 & 131 & $70(16.55)$ & 49 & 38 & $35(13.42)$ \\
$2 \times 2$ & 272 & 132 & $81(14.05)$ & 49 & 40 & $38(13.42)$ \\
$4 \times 1$ & 270 & 130 & $73(15.30)$ & 49 & 39 & $36(12.80)$ \\
$8 \times 1$ & 271 & 131 & $83(13.58)$ & 51 & 45 & $44(10.92)$ \\
$4 \times 4$ & 272 & 131 & $91(12.48)$ & 50 & 44 & $44(8.42)$ \\
$8 \times 8$ & 274 & 132 & $109(10.30)$ & 58 & 59 & $56(5.77)$ \\
\hline
\end{tabular}

Table 1. Number of iterations for $T=1$ using the old $\left(p^{*}=3.77\right)$ and new $\left(p^{*}=8.42\right)$ theoretically optimized parameters compared to the best choice (given in parentheses after the iteration number) obtained by numerical minimization of the iteration number

the type of decomposition: $8 \times 1$ means for example a one dimensional, banded decomposition into 8 subdomains. Clearly the new estimate of $p^{*}$ leads to a significantly better method, and the iteration number is now much closer to the best possible in the algorithm. We also see that the numerically best parameter depends on the number of subdomains, a fact which our analysis based on two subdomains can not capture. The iteration number then grows with the number of subdomains for larger time windows without coarse grid, as in the case of the classical Schwarz waveform relaxation algorithm, see [9].

Next, we present a large scale study in Table 2 , where $\Omega$ is decomposed into bands, $2 \times 1,3 \times 1,4 \times 1 \ldots$, for various length of the time interval. We observe how the convergence is independent of the number of subdomains for the theoretically optimized parameter, a result that has been proved for classical Schwarz waveform relaxation methods over short time intervals in [9], see also [3], but no analytical results exist so far for optimized Schwarz methods. We also see again that the new optimized parameter performs as well as the numerically optimized one when GMRES is used. We show in Figure 1 the convergence curves of the Schwarz waveform relaxation algorithm used as an iterative solver and as a preconditioner for GMRES, both for the the- 

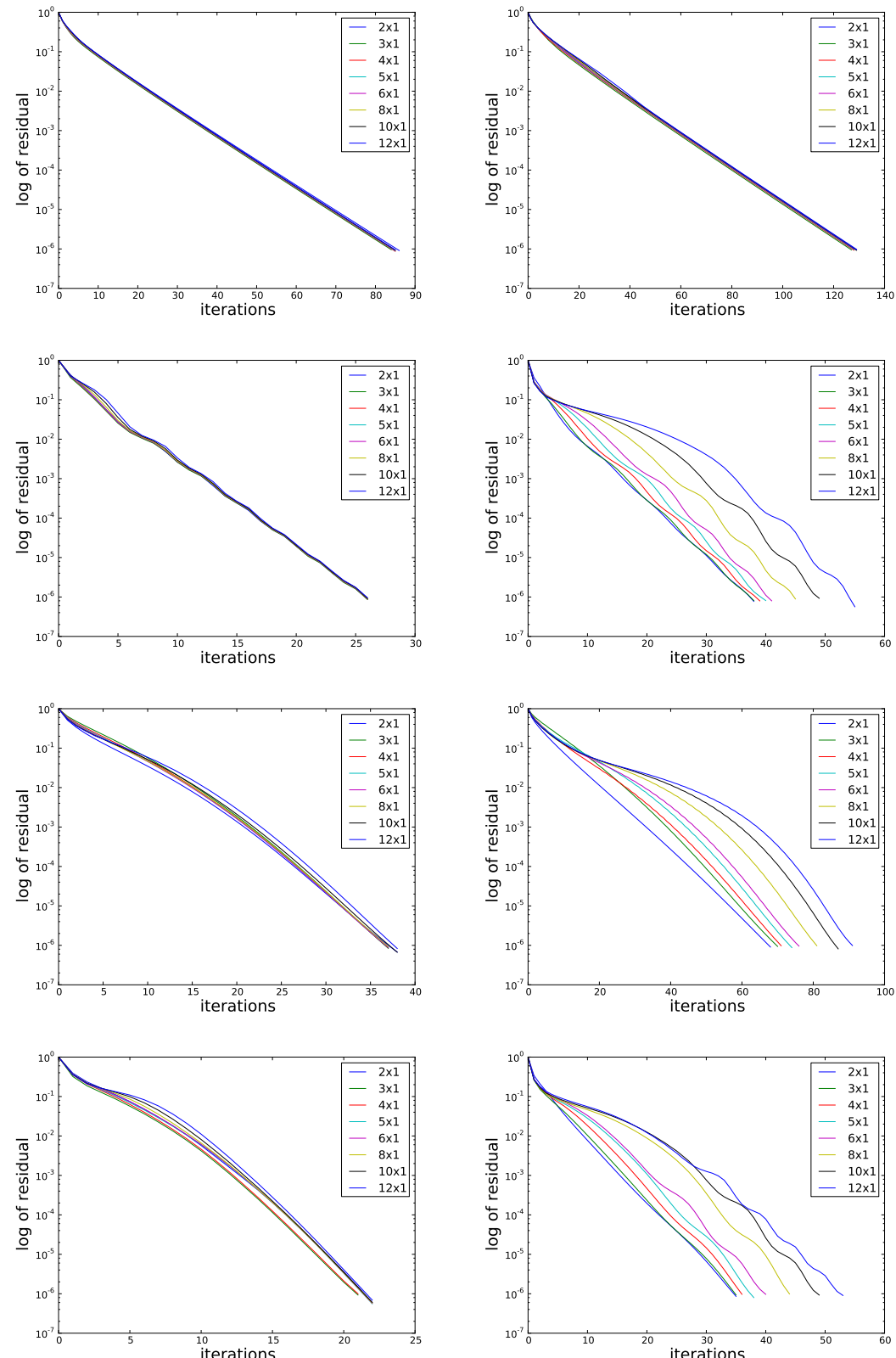

Fig. 1. Convergence behavior with the theoretically optimized parameter $p^{*}$ in the top four pictures, and the numerically optimized one below: for $T=\frac{1}{20}$ on the left and $T=1$ on the right using Schwarz as an iterative method in the first and third row, and as preconditioner for GMRES in the second and fourth row 


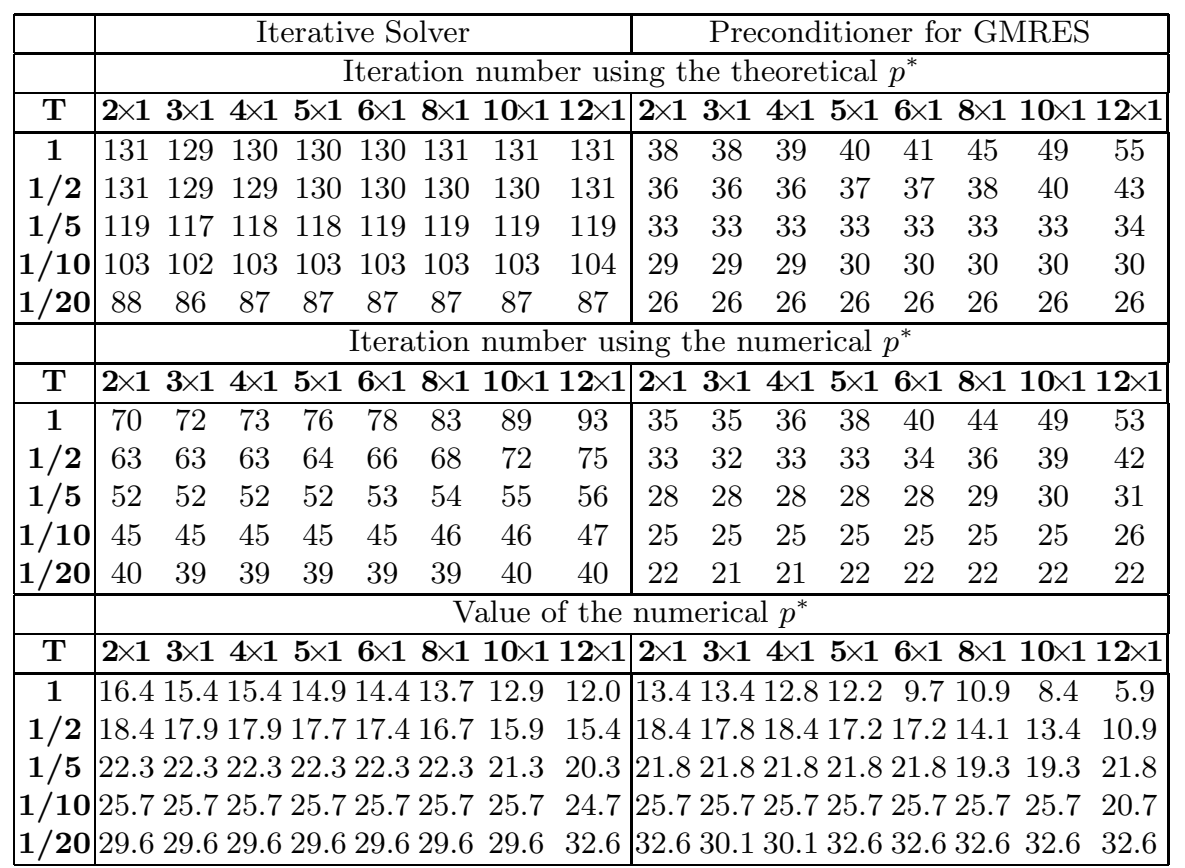

Table 2. Number of iterations for different final times $T$ and different numbers of subdomains, both using the theoretical and numerically optimal $p^{*}$, which was $p^{*} \in$ $\{8.4,8.4,9.3,10.7,12.6\}$ for $T \in\left\{1, \frac{1}{2}, \frac{1}{5}, \frac{1}{10}, \frac{1}{20}\right\}$, and the value of the numerically optimal $p^{*}$

oretically and numerically optimized parameter. We see that the algorithm's convergence behavior does not depend on the number of subdomains over the short time interval. We also observe that the numerically optimized parameter leads to a superlinear convergence regime, while the theoretical one gives a linear convergence regime.

We next turn to the case of decompositions with cross points, where the square domain $\Omega$ is decomposed into smaller square or rectangular subdomains, $2 \times 2,3 \times 2,3 \times 3, \ldots$. The results are given in Table 3 . The algorithm performs similarly to the banded case, but an interesting new observation is that the number of iterations over the long time interval is constant over antidiagonals in the table (notice the numerically optimized case in particular), which shows that the diameter of the graph of the decomposition is relevant for the dependence on the number of subdomains over long times.

We finally show a numerical experiment for $T=1$ on the geometrically challenging decomposition shown in Figure 2 on the left, which our generic simulator, implemented in Python using MPI, can easily handle. The computational mesh is twice refined from the mesh shown, and the convergence history with GMRES is shown in Figure 2 on the right. 
OSWR: A Large Scale Numerical Study

\begin{tabular}{|c|c|c|c|c|c|c|c|c|c|c|}
\hline \multirow[t]{2}{*}{$T$} & \multicolumn{5}{|c|}{ Iterative solver } & \multicolumn{5}{|c|}{ Preconditioner for GMRES } \\
\hline & $x$ & 3 & 4 & 5 & 6 & 2 & 3 & 4 & 5 & 6 \\
\hline \multirow{5}{*}{$\frac{1}{20}$} & $288(45)$ & & & & & $\overline{228(25)}$ & & & & \\
\hline & $387(45)$ & $88(45)$ & & & & $328(25)$ & $28(25)$ & & & \\
\hline & $488(45)$ & $87(45)$ & $87(46)$ & & & $428(25)$ & $29(26)$ & $29(25)$ & & \\
\hline & $588(45)$ & $88(46)$ & $88(46)$ & $88(46)$ & & $528(25)$ & $29(26)$ & $29(26)$ & $29(25)$ & \\
\hline & $688(45)$ & $87(46)$ & $88(46)$ & $88(46)$ & $88(46)$ & $628(25)$ & $29(26)$ & $29(26)$ & $29(25)$ & $29(25)$ \\
\hline \multirow{5}{*}{$\frac{1}{5}$} & $2 \mathbf{2 1 2 0 ( 6 0 )}$ & & & & & $235(32)$ & & & & \\
\hline & $3119(60)$ & $119(61)$ & & & & $335(32)$ & $35(32)$ & & & \\
\hline & $4119(61)$ & $119(61)$ & $120(61)$ & & & $435(32)$ & $35(32)$ & $35(32)$ & & \\
\hline & $5119(61)$ & $119(61)$ & $120(62)$ & $120(62)$ & & $535(32)$ & $35(32)$ & $35(32)$ & $35(32)$ & \\
\hline & $6119(62)$ & $119(62)$ & $119(62)$ & $120(63)$ & $120(64)$ & $635(33)$ & $35(33)$ & $35(32)$ & $35(33)$ & $35(33)$ \\
\hline & $2 \mathbf{2 1 3 2 ( 8 1 )}$ & & & & & $240(38)$ & & & & \\
\hline \multirow{4}{*}{1} & 3 131(84) & $131(86)$ & & & & $341(40)$ & $42(41)$ & & & \\
\hline & $4131(86)$ & $131(89)$ & 131(91) & & & $442(41)$ & $43(43)$ & $44(44)$ & & \\
\hline & 5 131(88) & 131(91) & 131(93) & 131(96) & & $543(43)$ & $44(44)$ & $46(45)$ & $48(47)$ & \\
\hline & 6 131(91) & 131(93) & 131(96) & 131(98) & 131(100) & $645(45)$ & $46(45)$ & $48(47)$ & $50(49)$ & $52(51)$ \\
\hline
\end{tabular}

Table 3. Number of iterations for the theoretical optimized parameter, and in parentheses for the numerically optimized one for different final times $\mathrm{T}$ and different decompositions with cross points

\section{Conclusions}

We presented new theoretical estimates for the parameters in the optimized Schwarz waveform relaxation algorithm. Our large scale numerical study shows that the new parameters perform significantly better than the old ones, and they reveal properties of the algorithm which are not yet understood theoretically, like the good scaling properties when the number of subdomains is increased, or the dependence of the parameter on the number of subdomains.

Acknowledgments: This work was partially supported by the Swiss SNF grant 200020-121561/1, and by the French ANR projects COMMA and SHP$\mathrm{CO} 2$.
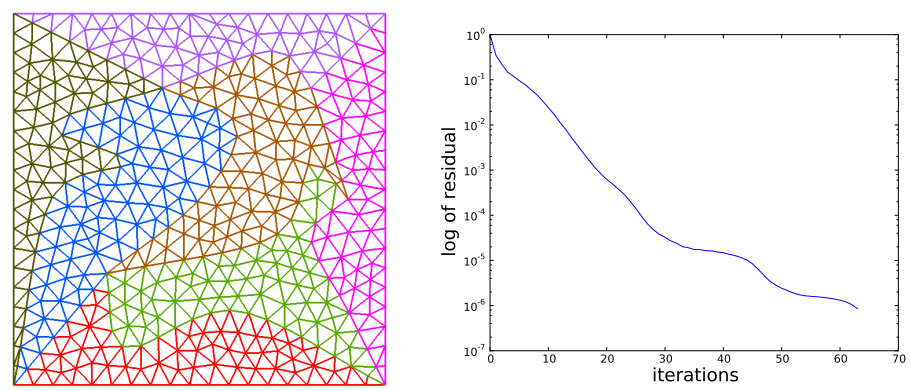

Fig. 2. Challenging geometrical decomposition on the left, and convergence curve on the right 


\section{References}

[1] Daniel Bennequin, Martin J. Gander, and Laurence Halpern. A homographic best approximation problem with application to optimized Schwarz waveform relaxation. Math. of Comp., 78(265):185-232, 2009.

[2] Morten Bjørhus. On Domain Decomposition, Subdomain Iteration and Waveform Relaxation. PhD thesis, University of Trondheim, Norway, 1995.

[3] D.S. Daoud and M.J. Gander. Overlapping schwarz waveform relaxation for advection reaction diffusion problems. Bol. Soc. Esp. Mat. Apl., 46:75-90, 2009.

[4] Martin J. Gander. Optimized Schwarz methods. SIAM J. Numer. Anal., 44(2):699-731, 2006.

[5] Martin J. Gander. On the influence of geometry on schwarz methods. In Nineteenth international Conference of Domain Decomposition Methods. Springer Verlag, 2010. Submitted.

[6] Martin J. Gander and Laurence Halpern. Optimized Schwarz waveform relaxation methods for advection reaction diffusion problems. SIAM J. Numer. Anal., 45(2):666-697, 2007.

[7] Martin J. Gander, Laurence Halpern, and Frédéric Nataf. Optimal convergence for overlapping and non-overlapping Schwarz waveform relaxation. In C-H. Lai, P. Bjørstad, M. Cross, and O. Widlund, editors, Eleventh international Conference of Domain Decomposition Methods. ddm.org, 1999.

[8] Martin J. Gander and Andrew M. Stuart. Space time continuous analysis of waveform relaxation for the heat equation. SIAM J., 19:2014-2031, 1998.

[9] Martin J. Gander and Hongkai Zhao. Overlapping Schwarz waveform relaxation for the heat equation in n-dimensions. BIT, 42(4):779-795, 2002.

[10] Eldar Giladi and Herbert B. Keller. Space time domain decomposition for parabolic problems. Numerische Mathematik, 93(2):279-313, 2002.

[11] Laurence Halpern. Optimized Schwarz waveform relaxation: roots, blossoms and fruits. In Eighteenth international Conference of Domain Decomposition Methods. Springer Verlag, 2009.

[12] Veronique Martin. An optimized Schwarz waveform relaxation method for unsteady convection diffusion equation. Applied Numerical Mathematics, 52(4):401-428, 2005.

[13] Virginia Torczon. Multi-Directional Search: A Direct Search Algorithm for Parallel Machines. PhD thesis, Department of Computational and Applied Mathematics, Rice University, Houston, 1990.

[14] Binh Minh Tran. Schwarz Waveform Relaxation Methods. PhD thesis, LAGA, Paris 13, 2010. In preparation. 